\section{ALGUNAS CONSIDERACIONES TEÓRICAS EN TORNO A LA FAUNA COMO INDICADORA DE ESPACIOS AGRARIOS EN LA PREHISTORIA}

\author{
THEORETICAL REMARKS ON THE \\ FAUNA AS AN INDICATOR OF \\ AGRICULTURAL LANDSCAPES IN \\ PREHISTORIC TIMES
}

\section{ARTURO MORALES MUÑIZ (*)}

\section{RESUMEN}

La calidad bioindicadora de un taxón viene determinada por el grado de asociación con determinadas variables ambientales y no por otras circunstancias tales como su abundancia o tamaño. Desde esta perspectiva y desde el punto de vista del análisis del espacio agrario como un ecosistema de creciente importancia en la biosfera, el presente estudio analiza algunas de las circunstancias que permiten evaluar la calidad, como bioindicadores, de dos grupos de fauna bien diferentes, las aves y las garrapatas. El trabajo pretende asimismo sentar las bases de cara a una concepción más integradora y equilibrada de la arqueozoología cono ciencia pluridisciplinar.

\section{ABSTRACT}

The value of a taxon in terms of bioindication depends more on its degree of association with a series of environmental parameters than on other circumstances such as its size or abundance. From this standpoint and considering agricultural spaces as ecosystems of increas-

(*) Laboratorio de Arqueozoología. Departamento Biología. Universidad Autónoma de Madrid. Cantoblanco. 28049 Madrid.

$\mathrm{El}$ artículo fue remitido en su versión final el 20-V-96. ing importance on the biosphere, we analyze in this paper some of the features which allow one to evaluate the quality, as bioindicators, of two very different faunal groups, namely birds and mites. Another aim of this work is to stress the pluridisciplinar and holistic nature of archaeozoology.

Palabras clave: Fauna. Aves. Ácaros. Bioindicador. Agricultura. Prehistoria.

Key words: Fauna. Birds. Mites. Bioindication. Agriculture. Prehistory.

\section{INTRODUCCIÓN}

Los espacios agrarios constituyen el biotopo de más reciente aparición sobre la biosfera con apenas diez milenios de existencia. A pesar de ello las áreas cultivadas ocupan en la actualidad una parte importante de la superficie terrestre, tanto por su extensión (aproximadamente $40 \%$ de la tierra emergida) como por la riqueza de los suelos que ocupan (Whittaker \& Likens, 1975). 
Aunque los espacios agrarios varían enormemente en su fisonomía según épocas y zonas geográficas, características botánicas, etc., e incluyen paisajes tan diferentes como las dehesas, huertos, jardines, arrozales, barbechos o campos de cereales, existe una serie de características que les resultan comunes. Entre éstas las más notables serían las siguientes:

1. Energéticamente hablando su existencia depende, parcial o totalmente, de la actividad antrópica continuada (entendida ésta en sentido amplio, es decir, incluyendo también la de los animales domésticos) que asegura una inversión de esfuerzo considerable (abono, pastoreo, sembrado, etc.) ajena con frecuencia al propio ecosistema.

2. El grado de complejidad estructural es muy inferior al de la mayoría de los ecosistemas no antrópicos. En general, los ecosistemas agrarios suelen enfatizar la productividad a base de asegurar que la mayor parte de la energía invertida podrá ser recuperada directa (consumo) o indirectamente (usos secundarios) por el hombre. Ello supone con frecuencia eliminar toda la estructura "superflua" (en términos de aprovechamiento) de un ecosistema maduro como puede ser la estratificación, las cadenas tróficas dilatadas o la distribución en parche de taxones. Todo ello con el fin de crear un sistema homogéneo, pobre en diversidad y con alta tasa de renovación que pueda ser explotado regularmente y con el mínimo esfuerzo posible. El paradigma de esta estrategia serían los monocultivos intensivos (por ejemplo, campos de cereales) en donde la energía de prácticamente toda la comunidad herbácea es aprovechada exclusivamente por el hombre o por sus animales domésticos (al menos desde una perspectiva teórica). Tales sistemas, como ha quedado sobradamente demostrado, suelen ser enormemente frágiles, costosos de mantener y, en general, de vida muy limitada con todas las repercusiones que para la población humana tales características conllevan.

3. En cualquier caso, los espacios agrarios son ecosistemas que "impone" la actividad humana en una zona a costa de sacrificar comunidades preexistentes en mayor o menor grado y con mayor o menor fortuna.
Básicamente, existen cuatro modalidades a través de las cuales el hombre es capaz de crear nuevos hábitats en una zona determinada: 1) incendios; 2) sendas; 3) acúmulos de desechos y aparición de áreas con alto contenido de nitrógeno; y 4) creación de suelos "abiertos" (es decir, desprovistos de cobertura vegetal). La primera de estas modificaciones es posiblemente la más antigua y violenta forma de alteración del llamado "orden natural" (Anderson, 1956). Sin duda constituyó un modo importante de modificación de comunidades bióticas desde el Paleolítico y ha podido ser documentado tanto en culturas agrícolas como de cazadores-recolectores (Anderson, 1956; Iversen, 1956).

En contraste con esta situación, la creación de suelo "abierto", como actividad antrópica regular, pertenece en mucha mayor medida que el incendio a la etapa agrícola de la humanidad. En la mayor parte de las áreas templadas, subtropicales o tropicales, el suelo desprovisto de vegetación - la tierra- no constituye parte del "orden natural": la mayoría de la flora de estas regiones no germina en la tierra expuesta o, si germina, no suele madurar. Parece que esta actividad de apertura de suelos discurre íntimamente asociada con la consolidación del sedentarismo y es a partir de la misma cuando podemos atestiguar la magnificación de la acción moduladora del entorno por parte del hombre (Simmons, 1982). Es en este entorno, sedentario en mayor o menor medida en una primera etapa, donde se consolida el sistema de producción agropecuario aunque el orden de ambos agentes (agricultura y ganadería) y el momento de la consolidación del sistema productivo varíen de zona en zona y continúen siendo motivo de debate (Maisels, 1990).

De una u otra forma el sedentarismo supone, en palabras de Bernis (1988: 8), la aparición en la biosfera “... de pústulas destructoras rodeadas de un halo más o menos amplio sujeto a modificación". Tal concepto implica la existencia de un gradiente de biotopos entre el entorno natural y el protonúcleo antrópico sobre el cual actuarán diversos factores creando diferentes limitantes a los que responderán todos los organismos, tanto domésticos como no (Tchernov, 1992: figura 1). Sobre la base de la adaptación a los ambientes diferencialmente

T. P., 53, n. ${ }^{\circ} 2,1996$ 
antropizados reposa la condición bioindicadora de la fauna y flora en la actualidad.

\section{LA FAUNA AGRARIA: ACLARACIONES PREVIAS}

Diez milenios de existencia de espacios agrarios son, en términos geológicos, una fracción temporal despreciable a efectos de evolución de fauna (¡aunque no de flora!, véase Anderson, 1956). Si las características de estos espacios agrarios fuesen radicalmente diferentes de las existentes en otros ecosistemas terrestres, la única especie "agraria" en estos momentos sería el Homo sapiens.

Sin embargo, muchas de las características fisionómicas de los espacios agrarios duplican las encontradas en numerosos biotopos naturales (por ejemplo, campos de cereales y estepas, dehesas y sabanas, arrozales y márgenes de ríos, setos y sotobosques, etc.). Por esta razón, y debido a que gran cantidad de animales responde en mayor medida a cambios generales en la estructura de los ecosistemas que a variaciones microflorísticas o microtopográficas específicas de los mismos, un nada despreciable número de especies ha sido capaz de colonizar los espacios agrarios.

El caso de las aves, por ejemplo, es uno de los mejor conocidos. Las aves "... responden ante todo al relieve del terreno y a la fisonomía de la vegetación, y ello en gran parte por los condicionantes que ambos imponen a la conducta reproductora. En este sentido, podemos decir que aves esteparias son aquéllas propias de terrenos llanos, desarbolados y sin matorral alto..." (Juana, 1989: 201). No es de extrañar, por tanto, que tanto si la falta de árboles o matorral es debida a condiciones naturales (por ejemplo, aridez) como a la intervención humana, las especies que colonicen ambos tipos de ecosistemas sean similares, cuando no idénticas. En este sentido, cabe resaltar cómo la respuesta convergente de la avifauna a tales biotopos ha impedido zanjar la secular polémica sobre el carácter natural o antrópico de las estepas ibéricas (Juana, 1989).

Por otra parte, los espacios agrarios gozan de una serie de alicientes a efectos de colonización que, aunque sea de pasada, resulta conve-

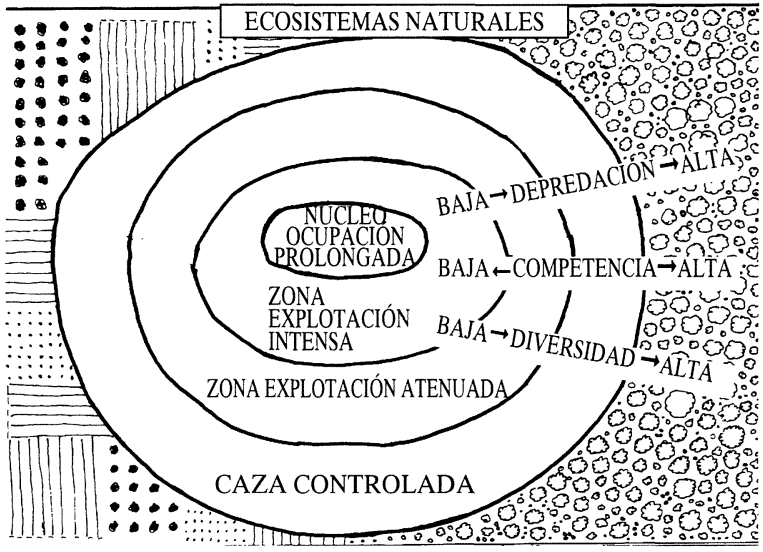

Fig. 1. Bandas ecológicas centrípetas surgidas alrededor de un asentamiento humano como consecuencia de una ocupación antrópica extensiva y prolongada así como por la explotación de los recursos circundantes, provocando una reversibilidad de los procesos ecológicos. El agotamiento mantenido de los recursos biológicos alrededor de los asentamientos humanos creó un peculiar y aislado miniecosistema prácticamente desprovisto de plantas y animales, al tiempo que abrió nuevas oportunidades (es decir, nichos) a especies, colonizadoras preadaptadas a su explotación (tomado de Tchernov, 1992).

niente recordar (Morales, 1989; Tchernov, 1992) (Fig. 1):

1. En primer lugar, el carácter "novedoso" dentro de la zona donde aparecen hace que los espacios agrarios se comporten, en una primera etapa, como "islas" recién emergidas, listas para ser colonizadas. La inicialmente baja diversidad de especies supone unos menores niveles de competencia interespecífica que constituye un indudable atractivo para organismos procedentes de ecosistemas maduros en donde apenas si existe espacio ecológico (es decir, nichos libres o recursos energéticos sin explotar).

2. En segundo lugar, la gestión antrópica del espacio agrario supone, entre otros factores, la práctica desaparición de depredadores (carnívoros, aves de presa, serpientes, etc.) en las áreas "gestionadas" por el riesgo que para el hombre suponen. Para muchos animales, en especial pequeñas aves, roedores y ciertos insectos, la desaparición (o relajación) de esta predo-presión constituye un importante aliciente para colonizar zonas de cultivo.

3. En tercer lugar, la propia dinámica del agrosistema resulta en sí misma atractiva para 
multitud de especies. En particular, la existencia de biotopos en donde el énfasis del esfuerzo radica en maximizar la productividad primaria constituye un poderoso acicate en la medida en que la disponibilidad trófica es uno de los factores determinantes de la distribución espaciotemporal de la fauna. La producción de granos, semillas y frutos de los espacios agrarios resulta, por ejemplo, determinante para comprender la abundancia de fauna edáfica en ellos, la cual, a su vez, determina la existencia de una amplia gama de vertebrados insectívoros, omnívoros y fitófagos. Si a esto le añadimos el componente marcadamente estacional de la producción, en no pocas ocasiones complementario, cuando no contrapuesto, con la producción de alimentos en ecosistemas naturales, completaremos una panorámica de indudable interés para la subsistencia de numerosos taxones. Una última matización en este contexto es que, si bien el hombre intenta aprovechar la totalidad de la biomasa potencialmente consumible en cualquier cultivo, parte de ésta permanece siempre en la zona aún después de la cosecha y constituye una fuente de alimento prioritario para muchas especies.

4. Por último, no debemos olvidar que la demografía humana en constante aumento ha supuesto el progresivo reemplazo de los ecosistemas naturales por agrosistemas, con frecuencia concentrando éstos en los suelos y zonas más fértiles de la biosfera. Desde esta perspectiva podemos comprender que existan fuertes presiones selectivas incitando a la disyunción ecológica en numerosas especies animales.

Aunque la mayoría de los animales no ha sido estudiada desde este punto de vista de adaptación al medio agrario, algunos autores han realizado clasificaciones de las faunas de acuerdo con su grado de asociación a biotopos antrópicos (Iankov, 1983; Boev, 1993). Distinguimos así:

1. Sinantrópicas estacionales: crían fuera de los espacios agrícolas y urbanos aunque visitan éstos durante ciertas épocas del año debido a razones tróficas o microclimáticas.

2. Sinantrópicas pasivas: crían en biotopos silvestres aunque algunos individuos pueden hacerlo en hábitats antrópicos análogos a éstos.

3. Sinurbanistas incipientes: especies en donde un sector importante de las poblaciones (se habla de hasta el $25 \%$ del total) invade los núcleos urbanos para criar.

4. Sinurbanistas avanzados: especies que, a fuerza de criar sistemáticamente alrededor de asentamientos humanos, acaban estableciendo poblaciones segregadas de las silvestres.

5. Sinurbanistas extremas: especies en donde la casi totalidad, cuando no la totalidad misma, de las poblaciones cría en asentamientos humanos, incluyendo los altamente urbanizados.

Aunque esta clasificación se ha basado fundamentalmente en información proporcionada por las aves, creemos que resulta extrapolable, por lo operativa, a cualquier otro grupo de fauna. Quizás el punto más interesante sea destacar como las divisiones no se establecen sobre unos criterios absolutos sino basándose en porcentajes populacionales. Ello, entre otras cosas, evidencia el carácter eminentemente dinámico y progresivo de la disyunción ecológica que se va operando en las especies silvestres al ser sometidas a presión por parte de la actividad humana.

Para nuestros propósitos, y dado que la fauna urbana viene condicionada por una distinta incidencia de las variables que consideraremos, las dos primeras categorías constituirían el objetivo primordial de análisis.

\section{LA FAUNA AGRARIA: ¿REALIDAD O IDEALIZACIÓN?}

La caracterización de una agrupación faunística como bioindicadora de paisaje agrario reposa, ante todo, sobre bases de inferencia analógica (Baird, 1989). Tales bases sólo pueden consolidarse tras estudios exhaustivos y concluyentes, algo que casi nunca ocurre. En efecto, mientras que el carácter bioindicador de la mayoría de los taxones de invertebrados resulta, en el mejor de los casos, vagamente conocido, en aquellos grupos de vertebrados que sí han sido objeto de investigación sistemática desde esta perspectiva, los resultados no dejan de ser con frecuencia ambiguos (Fig. 2). Por otra parte, la validez bioindicadora de una taxocenosis descansa sobre bases estadísticas am-

T. P., 53, n. ${ }^{\circ} 2,1996$ 

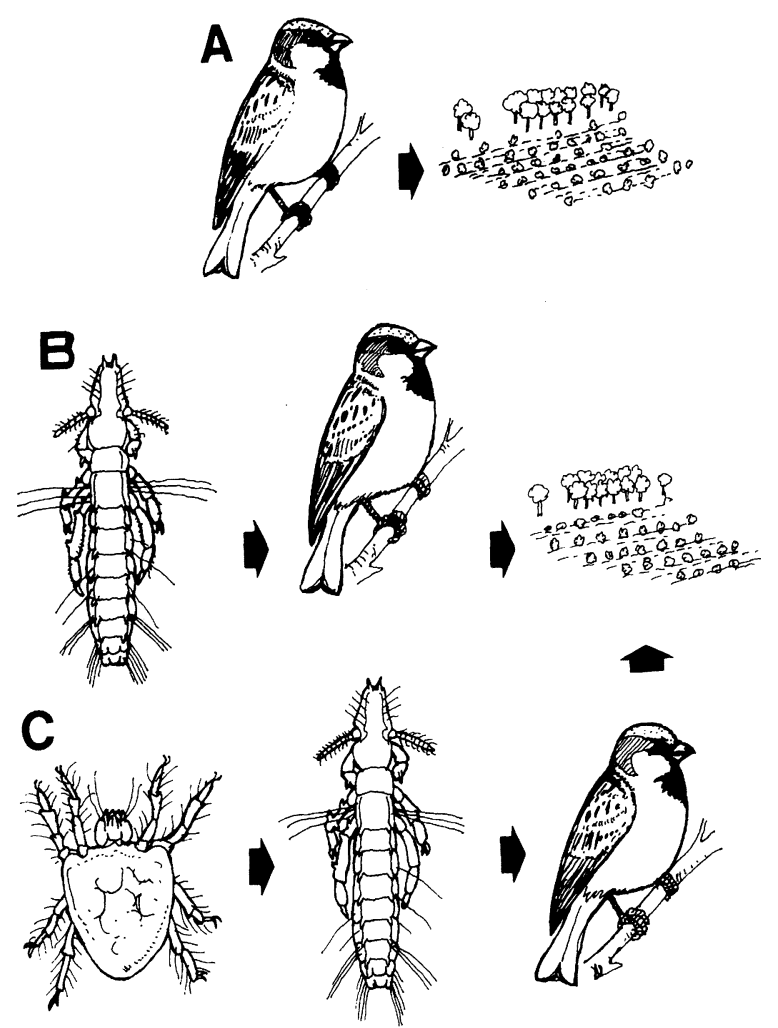

información resulta extrapolable al pasado. No podemos olvidar que partimos de circunstancias peculiares en donde la acción antrópica moduladora del entorno ha alcanzado niveles tales que, en mayor o menor medida, han alterado irreversiblemente los biotopos originales de gran número de especies. En esta "pseudobiosfera" o "antroposfera" los hábitos de muchas especies pueden perfectamente ser pálidos, cuando no equívocos, reflejos de circunstancias pretéritas.

La consideración de estas circunstancias, no debe ser, a pesar de todo, excusa para desestimar el análisis del tema aunque ello obligue a una profunda reflexión tendente a rechazar cualquier atisbo de dogmatización o simplificación relativo al carácter bioindicador de ciertos taxones.

Para ilustrar algunas de las cuestiones teóricas antes referidas, expondremos muy someramente el caso de dos grupos faunísticos muy diferentes en cuanto a conocimiento y papel bioindicador que, sin embargo, revisten un enorme interés desde el punto de vista arqueozoológico.

\section{LAS AVES}

Fig. 2. La bioindicación faunística puede ser directa (A: gorrión asociado con asentamientos humanos) o indirecta, la cual, a su vez, puede revestir diversos grados de complejidad. Así, en B, la presencia de un piojo malófago del gorrión, indirectamente evidencia la existencia de un paisaje antrópico en un yacimiento, mientras que en $\mathrm{C}$, se trata de un ácaro (garrapata) específico del malófago el que evidencia tal paisaje. Obviamente, la calidad bioindicadora de cualquier especie depende directamente del grado de asociación con determinados elementos (bióticos o abióticos) relacionados con dicho espacio antrópico. Por otra parte, añadir eslabones a una "cadena bioindicadora" como la representada disminuye su calidad en la medida en que incrementa el grado de incertidumbre implícito en la evaluación.

plias y obliga a disponer de muestras, sino abundantes, cuando menos aceptablemente diversificadas. Desde tal perspectiva, el aforismo "una golondrina no hace verano" resulta particularmente ilustrativo.

Por encima de estas limitaciones se sitúa la posible invalidez de cualquier base de inferencia analógica obtenida con datos actuales debido a la imposibilidad de calibrar en qué medida tal

Las aves constituyen, sin duda alguna, el grupo animal mejor conocido a excepción hecha de la fauna doméstica. Además de la fiabilidad que el conocimiento de su biología implica, se trata de un grupo diversificado, taxonómica y ecológicamente, cuya identificación osteológica suele ser con frecuencia más que aceptable (Baird, 1989; Morales, 1993). Ello asegura, en principio, un alto valor como bioindicadores, en especial a efectos del prehistoriador.

Lo cierto es que no todo son ventajas. Las aves, como animales voladores, tienen una gran capacidad de movimiento. Numerosas especies realizan migraciones. Esto implica que, a lo largo de un ciclo fenológico, pueden frecuentar diferentes zonas y biotopos. Normalmente, las migraciones implican disyunciones de hábitos ecológicos, especialmente tróficos. El caso de los estorninos (insectívoros en la Europa atlántica donde crían y azote de nuestros olivares durante la invernada) no es un caso aislado. 
Por encima de ello, como antes comentamos al hablar de aves esteparias, las aves responden a un conjunto de factores del entorno (topografía, fisionomía vegetal, etc.) que pueden haber sido producidos de modo convergente por diversos agentes, entre ellos el hombre.

Tomemos el caso de la avifauna denominada agrícola (Tablas 1 y 2). Si bien tenemos una idea clara, a priori, de cuáles son las especies que encontraremos en zonas cultivadas (por ejemplo, codorniz, alondra, calandria, etc.), lo que no todos sabemos es que esta avifauna agrícola depende en no poca medida:

a) Del entorno natural original sobre el que se asienta el paisaje agrario.

b) Del tipo de cultivo y el grado de intensidad con el que se practica en una zona dada.

Como ejemplo del primer punto, O'Connnor \& Schrubb (1986) demuestran cómo la avifauna agrícola en Inglaterra se encuentra mayoritariamente constituida por especies forestales (tales como mirlos y pinzones) u, ocasionalmente, de orla de bosque (acentor común). De hecho, las 11 especies "agrícolas" más abundantes incluyen, si exceptuamos a la alondra y al escribano cerillo, a las 11 especies forestales más frecuentes (O'Connor \& Schrubb, 1986: 13; Tabla 1). Está claro que en tal situación a un arqueozoólogo le resultaría en extremo díficil decidir si una avifauna se corresponde con un paisaje agrario o forestal y esta caracterización en Inglaterra es extensiva a la mayoría de los sectores agrícolas de la Europa eurosiberiana incluyendo el Cantábrico.

Frente a ello, la avifauna de los espacios agrarios ibéricos se corresponde con elementos esteparios que posiblemente ya existían en la Península en el momento de introducción de la agricultura y que respondieron bien al aclaramiento y deforestación que aquella práctica introdujo (Tabla 1). En el caso de la avifauna reproductora asentada en los espacios agrarios estructuralmente menos complejos (es decir, las extensiones cerealistas), por ejemplo, encontramos comunidades dominadas por especies típicas del Mediterráneo semiárido (por ejemplo, terrera común, cogujada montesina, curruca carrasqueña, collalba rubia, etc.) (Suárez y Ramirez, 1987; Tellería y cols., 1988; Tabla 1). Esta aseveración es particularmente

\begin{tabular}{|c|c|c|}
\hline \multicolumn{3}{|c|}{ ESTEPAS NATURALES (COMUNIDADES) } \\
\hline \multicolumn{2}{|l|}{ MELANOCORYPHA } & ALAUDA \\
\hline \multicolumn{2}{|l|}{ Sisón } & Aguilucho Pálido \\
\hline \multicolumn{2}{|l|}{ Avutarda } & Perdiz Pardilla \\
\hline \multicolumn{2}{|l|}{ Alcaraván } & Guión de Codornices \\
\hline \multicolumn{2}{|l|}{ Ganga } & Avefría \\
\hline \multicolumn{2}{|l|}{ Ortega } & Bisbita Común \\
\hline \multicolumn{2}{|l|}{ Alondra Dupont } & Alondra Común \\
\hline \multicolumn{2}{|l|}{ Calandria } & Collalba Gris \\
\hline \multicolumn{2}{|l|}{ Terrera Común } & Limícolos (varias sp.) \\
\hline \multicolumn{3}{|l|}{ Terrera Marismeña } \\
\hline \multicolumn{3}{|l|}{ Cogujada } \\
\hline \multicolumn{3}{|l|}{ Bisbita Campestre } \\
\hline \multicolumn{3}{|l|}{ Collalba Gris } \\
\hline \multicolumn{3}{|l|}{ Collalba Rubia } \\
\hline \multicolumn{3}{|l|}{ Triguero } \\
\hline \multicolumn{3}{|c|}{ ESPACIOS AGRARIOS (TIPOS) } \\
\hline CEREALES & ERIALES & PRADOS \\
\hline Codorniz & Terrera Común & Mirlo (*) \\
\hline Alondra Común & Cogujada Montesina & Pinzón (*) \\
\hline Calandria & Alondra Dupont & Acentor Común (+) \\
\hline Terrera Común & Bisbita Campestre & Petirrojo (*) \\
\hline Cógujada Montesina & Curruca Tomillera & Chochín (*) \\
\hline Curruca Carrasqueña & & Alondra Común \\
\hline Collalba Rubia & & Herrerillo $(*)$ \\
\hline Tarabilla Común & & Zorzal Común (*) \\
\hline Triguero & & Escribano Cerillo (+) \\
\hline & & Mosquitero Musical $(*)$ \\
\hline & & Carbonero $(*)$ \\
\hline & & Pardillo (+) \\
\hline & & Verderón (+) \\
\hline & & Estornino Pinto \\
\hline & & Curruca Zarcera (+) \\
\hline
\end{tabular}

Tabla 1. Principales especies de aves en diversos espacios abiertos, tanto agrícolas como naturales (en cursiva especies estivales; en negrita especies cuyos efectivos locales se incrementan en invierno con migrantes eurosiberianos). La relación de prados incluye, por orden decreciente de importancia, las 15 especies más frecuentes en Inglaterra (datos que, en principio, resultan extrapolables a la mayoría de los sectores agrícolas eurosiberianos). Otros símbolos: $(*)$ origen forestal; $(+)$ origen orla de bosque (ecotono). Como puede observarse, muchas especies aparecen en varias categorías de espacios y ninguna puede asociarse con exclusividad a un biotopo. Por ello, la calidad como bioindicadores es muy limitada. Por otra parte, las cambiantes fenologías de la mayoría de los taxones suponen alteraciones de envergadura a lo largo del año en las distintas taxocenosis (tomado de O'Connor \& Schrubb, 1986; Juana, 1989).

cierta en el caso de los eriales, un medio xérico peculiar que contrasta con los húmedos prados eurosiberianos, y en donde se asienta la mayor parte de la avifauna esteparia ibérica (Valverde, 1958). 
Este "sustrato" estepario en la avifauna de los espacios agrícolas de la Iberia mediterránea, coexiste, en mayor o menor medida, con otros tipos de faunas acompañantes que hacen mucho más problemática la caracterización unívoca del entorno. Así, por ejemplo, los bosques de galería, sotos y setos albergan una avifauna de marcado carácter eurosiberiano forestal (por ejemplo, chochín, ruiseñor, mosquitero común, petirrojo, etc.) (Hernández y Alegre, 1991; Parra, 1982c), mientras que las especies acompañantes de avifauna esteparia abundan en los cultivos más abiertos (Tabla 2). Huelga decir que todas las categorías de sinurbanistas que antes hemos definido penetran con regularidad en los espacios agrícolas ampliando de este modo la lista de taxones potencialmente recuperables en los mismos. Por otra parte, en los espacios agrarios menos modificados (por ejemplo, dehesas) las diferencias avifaunísticas con espacios naturales son, a todos los efectos, imperceptibles (Parra, 1982a, b).

Un último aspecto sobre el que conviene volver a insistir es el referido a los ciclos estacionales que, lejos de corregir ambigüedades, con frecuencia complican la situación. Así, muchas de las especies caracterizadoras del paisaje agrario (por ejemplo, bisbitas, collalbas, codornices, currucas, etc.) son migrantes transaharianos que desaparecen durante las épocas inclementes del año (Tablas 1 y 2). Frente a éstos, muchas especies sedentarias ven sus efectivos incrementados durante otoño e invierno con la llegada masiva de poblaciones europeas. La mayoría de las invernantes pertenece al sector agrícola europeo que podríamos denominar "básico" (es decir, forestal) (Tabla 1) aunque durante la invernada frecuentan los biotopos agrícolas más abiertos, como baldíos, barbechales, etc. En esta situación encontraríamos, por ejemplo, a la mayoría de los fringílidos, en especial los pardillos. Tales cambios de abundancias y disyunciones de hábitos hacen que sea en extremo difícil una caracterización, no ya de taxones sino de toda una taxocenosis (actual o prehistórica), como paradigma de paisaje agrícola. Para complicar más aún las cosas, la propia fenología de muchas especies resulta ser en extremo dinámica. Así, tanto migrantes transaharianos como la codorniz o la cigüeña e inver-

\begin{tabular}{|lll|}
\hline RUPICOLA & ORLA BOSQUE & EDIFICACIONES \\
Alimoche & Pito Real & Cigïeña Común \\
Buitre Leonado & Totovía & Cernícalo Primilla \\
Cernícalo Común & Alcaudón Chico & Lechuza Común \\
Halcón Peregrino & Alcaudón Real & Abubilla \\
Paloma Bravía & Verdecillo & Vencejo Común \\
Paloma Zurita & Jilguero & Golondrina Común \\
Mochuelo & Pardillo & Estornino Negro \\
Abejaruco & & Gorrión Común \\
Carraca & & Gorrión Molinero \\
Collalba Negra & & \\
Cuervo & & \\
Grajilla & & \\
Chova Piquirroja & & \\
Gorrión Chillón & & \\
\hline
\end{tabular}

Tabla 2. Avifauna acompañante en las estepas ibéricas clasificada según hábitat de origen (negritas y cursivas como en la Tabla 1). Básicamente, la avifauna de origen urbano es avifauna rupícola (es decir, de roquedo). Dado que muchos de estos taxones pueden aparecer en diferentes tipos de medios, además de las estepas y sus biotopos de origen, su calidad bioindicadora de espacios agrarios es bastante pobre.

nantes como las agujas, se han convertido progresivamente en especies sedentarias en nuestro suelo durante los últimos años, en parte como consecuencia de las nuevas disponibilidades de alimentos que ha introducido la intervención humana (O'Connor \& Schrubb, 1986; Tellería y cols., 1988).

Completamos este poco edificante panorama diciendo que, de una forma general, la avifauna ibérica indicadora de espacios agrarios está fundamentalmente compuesta por paseriformes (en especial alaúdidos), aves cuya identificación osteológica resulta en extremo problemática en ausencia de determinados elementos diagnósticos (Morales, 1993). Todas estas limitaciones, unidas a las de carácter general comentadas al inicio de la sección, nos hace mantener serias reservas sobre la operatividad a efectos bioindicadores de un sector de fauna sobre el que, a priori, todas las cartas estarían a favor.

\section{LOS ÁCAROS}

Los ácaros, pequeños artrópodos emparentados con las arañas y colectivamente denominados garrapatas, constituyen un caso muy di- 

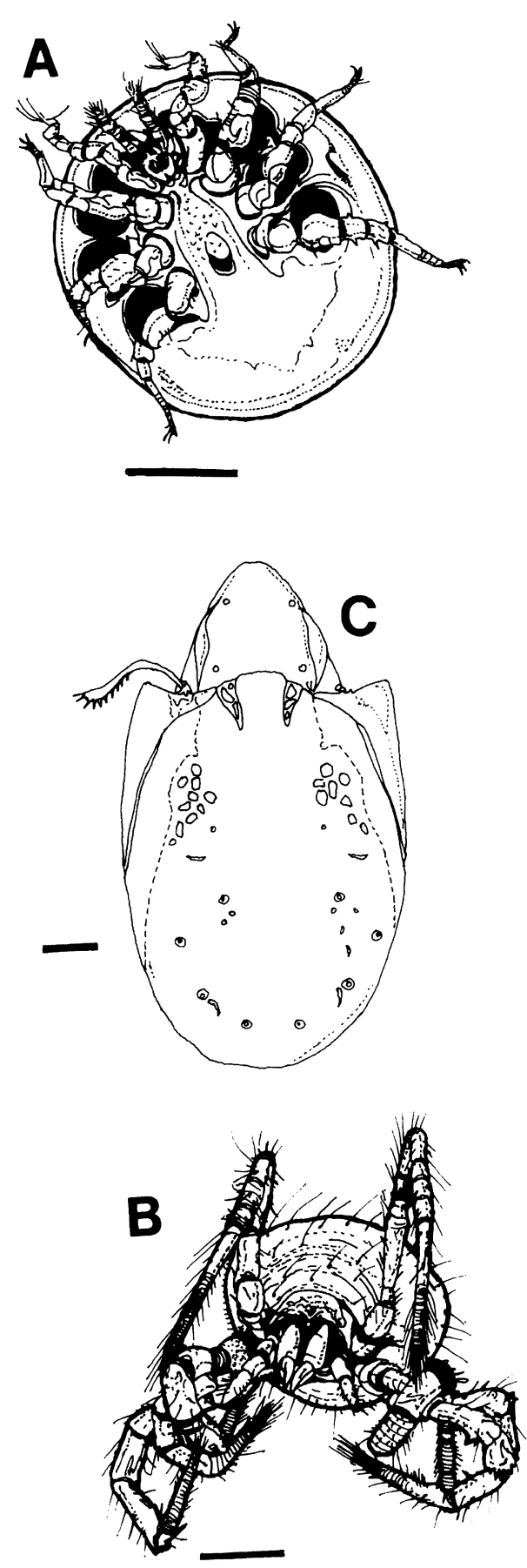

Fig. 3. Acaros edáficos de interés en la bioindicación de espacios agrarios. A. Uropoda (Cilliba) cassidea, un uropódido, grupo característico de suelos quemados. B. Pergamasus sp. género de gamasinos muy frecuente en excrementos de caballo. C. Xylobates capucinus, un oribátido recuperado en el yacimiento de Peñalosa (prov. Jaén; Edad del Bronce) (Morales y Sanz, 1994). Las escalas indican un milímetro. ferente al de las aves en lo que a posibilidades como bioindicadores se refiere (Morales \& Sanz, 1994).

Para empezar, se trata de un grupo ubicuo en prácticamente todos los ambientes del planeta, desde las trincheras oceánicas, y cimas de las cordilleras hasta biotopos antropogénicos (Schelvis, 1990, 1992, 1993). Contrariamente a lo que podría pensarse, la mayoría de las especies es de vida libre (Fig. 3).

Un segundo aspecto interesante es la aparentemente lenta tasa de diversificación morfológica del grupo, posiblemente como consecuencia de la estabilidad de sus nichos ecológicos (Krivolutsky \& Druck, 1986) habiéndose descrito individuos de la actual familia Ctenacaridae en el Devónico, hace más de 300 millones de años (Shear y cols. 1984). Esto, a efectos prácticos, significa que, para los "cortos" lapsos temporales con los que trabaja el prehistoriador, las especies actuales pueden muy bien servir en la identificación de ejemplares subfósiles.

Los ácaros suelen aparecer con frecuencia en grandes densidades en ambientes edáficos, lo cual asegura que muchos de ellos puedan acabar formando parte de las tafocenosis potencialmente recuperables por los excavadores (Schelvis, 1992, 1993). Su metodología de análisis, por otra parte, es bien conocida y, desde el punto de vista del prehistoriador, no difiere mucho en cuanto a forma de la llevada a cabo con pólenes.

Un cuarto punto de interés radica en que la capacidad de desplazamiento de estos animales en biotopos terrestres, por el hecho de ser ápteros, es francamente limitada y ello los convierte en buenos indicadores de condiciones locales.

Obviamente, no todo son ventajas en el estudio de este grupo. Así, un problema importante es que muchos ácaros no se encuentran lo suficientemente quitinizados como para perdurar durante largo tiempo en el sedimento (Morales y Sanz, 1994). Afortunadamente, tres de los principales grupos para los arqueozoólogos (oribátidos, gamásidos y uropódidos) no presentan tal tipo de limitación.

Con diferencia, la limitante fundamental con la que nos encontramos reside en la diversidad taxonómica del grupo (más de 50.000 es- 
pecies conocidas hasta la fecha) que obliga a disponer de especialistas en taxones concretos (géneros, familias, subórdenes, etc.) e imposibilita a un solo investigador dedicarse al análisis de todos los ácaros potencialmente recuperables en yacimientos. En este sentido, también debemos decir que la fauna acarológica de carácter antrópico (o asociada a biotopos antropogenizados) es una fracción reducida de la actualmente existente aunque, con todo, resulta absolutamente inabarcable para un solo especialista y obliga a recurrir al trabajo de equipos especializados.

El mayor incentivo para el estudio de ácaros en arqueozoología radica en su calidad como bioindicadores. En efecto, si bien conocemos actualmente muchas especies de amplia valencia ecológica, no menos cierto es que el grado de especificidad con el que muchas otras se asocian a determinados biotopos/nichos las convierte en óptimos objetos de estudio (Morales y Sanz, 1994; Gil-Martín y Subías, 1995). Por eso, con el desarrollo en profundidad de las investigaciones de carácter biocenótico y biogeográfico ocurrido en España durante la década de los ochenta surgió el germen de la utilización de los distintos taxones, no ya como diferenciadores de los medios bióticos sino como tipificadores de diferentes niveles o grados de alteración de los medios como consecuencia de las actividades humanas. Para ello se han utilizado tres grupos de ácaros, cada uno de los cuales permite el análisis de un tipo diferente de alteración (Schelvis, 1992; Morales y Sanz, 1994; Gil-Martín y Subías, 1995).

\section{Los oribátidos y los suelos agrícolas}

Los oribátidos, ácaros de exoesqueleto esférico bien quitinizado, constituyen uno de los grupos de fauna edáfica más abundante y selectiva. Su pequeño tamaño, al igual que ocurre con los otros grupos considerados, asegura que su presencia en sedimentos arqueológicos no se deberá a los caprichosos dictados de la selección intencionada por parte del hombre sino a circunstancias edáficas por mucho que éstas respondan también a la intervención humana (Schelvis, 1992).

\begin{tabular}{|ll|}
\hline ENCINAR NO ALTERADO & CAMPO CULTIVADO \\
Serratoppia serrata & Serratoppia minima \\
Medioppia media & Machuella entrisetosa \\
& Ramusella sengbuschi \\
SENDAS & Ja ieroppia cer us \\
Serratoppia minima & \\
Machuella entrisetosa & SUELO DE JUNCOS \\
OLMEDA & Ramusella puertomonttensis \\
Oxyoppioides decipiens & Corynoppia kosaro $i$ \\
Lauroppia similifallax & \\
\hline
\end{tabular}

Tabla 3. Ácaros oribátidos bioindicadores en diferentes biotopos del Monte de El Pardo (Madrid) (tomado de Subías y Mínguez, 1985).

Aunque la estrecha asociación entre determinados tipos de suelos y especies concretas de oribátidos había sido constatada desde hace años, no fué hasta que el conocimiento taxonómico se hizo lo suficientemente exhaustivo que se pudo constatar la calidad bioindicadora de estos artrópodos.

A través de análisis detallados de las asociaciones de oribátidos se ha podido constatar, en espacios limitados tales como el monte de El Pardo (prov. Madrid) cómo las especies permiten diferenciar los suelos básicamente inalterados del encinar en sí, de los suelos compactados que lo surcan a modo de vereda, o de los suelos agrarios (campos cultivados) que eventualmente se intercalan entre aquél (Subías y Mínguez, 1985; Tabla 3). Con frecuencia son especies de distintos géneros las que caracterizan un tipo de suelo mientras que las incluidas dentro de un mismo género suelen distribuirse preferencialmente sobre distintas condiciones edáficas dando la sensación de que existen microradiaciones adaptativas dentro de los géneros, dictando un proceso de especiación sobre variables ecológicas ligeramente modificadas. Incluso sobre suelos alterados, los oribátidos responden a otros parámetros del entorno, tales como pueden ser los gradientes de humedad en junqueras, olmedas, etc. (Subías y Mínguez, 1985; Subías y cols., 1985; Tabla 3).

Un problema interesante en la caracterización de espacios agrarios por parte de oribátidos es su calidad como bioindicadores en etapas de sucesión de suelos agrícolas. Así, Subías y cols. (1986) han sido capaces de tipificar la sucesión en eriales mediterráneos con lapsos de 


\begin{tabular}{|ll|}
\hline CULTIVADO & ABANDONADO +10 AÑOS \\
Verachthonius cf. laticeps & Scheloribates minifimbriatus \\
RECIÉN ABANDONADO & Phyllozetes emmae \\
(1-7 AÑOS) & Ramusella assimilis \\
Ja ieroppia cer us & Pseudoribates subsimillis \\
Bipassalozetes reticulatus & Hypogeoppia terricola \\
Zygoribatula frisiae & Scuto ertex sculptus \\
Eobrachychthonius oudemansi & Licnodamaeus costula \\
\hline
\end{tabular}

Tabla 4. Oribátidos bioindicadores sobre la sucesión de un erial de cultivo mediterráneo (tomado de Subías y cols., 1986).

abandono de hasta 40 años (Tabla 4) o la existencia de especies bioindicadoras para lapsos de abandono cortos (2-4 años) (Mahmud y cols., 1984; Tabla 5). Este fenómeno de bioindicación resultaría de amplia trascendencia en el estudio sistemático de espacios agrarios en la Prehistoria ya que el abandono temporal o definitivo de cultivos podría permitir confirmar o refutar hipótesis relativas a la pervivencia de culturas o asentamientos concretos en momentos de crisis. La sobrexplotación del medio y subsiguiente abandono podría abordarse con un análisis pedo-acarológico sistemático en zonas concretas.

El hecho de que muchos de estos estudios hayan sido iniciados en nuestro suelo (Arillo y cols., 1992) no impide reflexionar sobre el enorme grado de incertidumbre que aún poseemos sobre tales cuestiones como consecuencia del aún incipiente nivel de conocimiento de la fauna ibérica de oribátidos de la cual se estima que posiblemente la mitad esté aún pendiente de describir taxonómicamente (Subías, com. pers.). A pesar de ello, algunos de los usos cuyo efecto sobre suelos ha sido estudiado con oribátidos (por ejemplo, calidad y estructura del paisaje, irrigación, rotación de cultivos, manejo de malas hierbas, usos de setos, salinización de terrenos, etc.) sin duda acabarán encontrando, de la vía de especialistas interesados, su aplicación en el estudio de los paisajes agrícolas en el pasado.

\section{Los gamásidos y los depósitos de excrementos}

A diferencia de los oribátidos, los ácaros mesostigmáticos (Gamasida) son depredadores

\begin{tabular}{|ll|}
\hline CAMPO CULTIVADO DE VIÑA & CAMPO2 AÑOS DE ABANDONO \\
Passalozetes africanus & Multioppia wilsoni \\
& Scheloribates fimbriatus \\
CAMPO CULTIVADO DE CEBADA & \\
$\begin{array}{l}\text { Passalozetes agricola } \\
\text { Passalozetes africanus }\end{array}$ & CAMPO CON6 AÑOS \\
& DE ABANDONO \\
HORIZONTE INFERIOR DE & Zygoribatula connexa \\
CAMPO NUNCA CULTIVADO & HORIZONTE SUPERIOR \\
Passalozetes africanus & DE CAMPO NO CULTIVADO \\
Passalozetes agricola & Passalozetes agricola \\
\hline
\end{tabular}

Tabla 5. Especies dominantes de oribátidos en distintos medios agrícolas de la provincia de Toledo (tomado de Mahmud y cols., 1984).

que proliferan en medios ricos en materia orgánica entre los cuales las choriocenosis (biocenosis específica de las boñigas) resultan ser biotopos ideales (Mena y cols., 1990).

En realidad, la boñiga constituye un ecosistema en miniatura íntimamente ligado a determinados espacios agrarios (pastos, prados, etc.) los cuales, en buena medida, ha contribuido a formar. En efecto, la ineficiente capacidad digestiva de los ungulados hace que una sustancial proporción del alimento ingerido sea devuelto al suelo en forma de excrementos y permita el establecimiento de una coprofauna caracterizada por sucesiones biocenóticas destructivas que, unidas a las modificaciones del entorno, provocan la desintegración total de aquél. La boñiga es consustancial con el paisaje agrario en la medida en que absorbe calor aumentando la temperatura, capacidad de absorción y retención de agua del suelo subyacente. La coprofauna (sensu stricto) asegura, entre otros, un rápido reciclaje de la materia orgánica, su dispersión homogénea por el entorno y la conservación e incorporación al suelo del $80 \%$ del nitrógeno que dicha materia contiene.

Dado que la íntima asociación existente entre ganado doméstico y determinados espacios agrarios no puede inferirse a través del estudio directo de los excrementos, la choriocenosis constituye una valiosa herramienta de inferencia cuyas potencialidades apenas han sido exploradas (Schelvis, 1990). En este sentido, el estudio de los principales depredadores de esta comunidad, está resultando particularmente relevante. 
El reciente análisis llevado a cabo por Schelvis sobre acarofaunas en diferentes tipos de excrementos de fauna doméstica ha revelado la existencia de comunidades restringidas en cuanto a número de especies pero bioindicadoras del tipo de animal doméstico productor del excremento (Schelvis, 1992; Tabla 6). El estudio se llevó a cabo tanto en condiciones de estabulación como de pastoreo libre y las especies se mantuvieron constantes. Las potencialidades de confirmación de determinadas prácticas de pastoreo (vacuno en suelos más fértiles; ovicaprinos en suelos pobres) o de montanera (detección de ganado porcino en suelos de dehesas) en la prehistoria parecen confirmarse tras los análisis de este investigador en espacios rurales holandeses en el pasado (Schelvis, 1992). Al igual que en el caso de los oribátidos el futuro parece lleno de promesas.

\section{Los uropodinos y los suelos quemados}

El último grupo de ácaros de interés como bioindicadores de espacios agrícolas en la Prehistoria constituye uno de los conjuntos que antes colonizan los cenizales que se producen tras un incendio (Athias-Binche, 1987).

El fuego ha sido, antes lo mencionamos, la más antigua forma de manipulación del medio. Además de facilitar la deforestación, parece que la práctica de su uso pudo muy bien haber facilitado asimismo un tipo rudimentario de agricultura en la medida en que la ceniza contribuye a incrementar el ácido $\mathrm{PH}$ de muchos suelos forestales y elimina potenciales competidores para las plantas domésticas (Iversen, 1956; Simmons, 1982). De un modo o de otro, fuego y hombre parecen asociados desde el Pleistoceno medio y la quema sistemática de zonas continúa siendo aún hoy en día parte integral de las técnicas de gestión agrícola.

En este contexto cobra interés el uso de faunas de uropodinos quienes, al igual que ocurría con los oribátidos en suelos abandonados o los gamásidos en boñigas, se estructuran en una sucesión de comunidades colonizadoras que van penetrando progresivamente en suelos quemados prácticamente desde el momento en que se enfría la ceniza. El carácter bioindicador

\begin{tabular}{|ll|}
\hline OVEJA & VACA \\
Halolaelaps typo R21a $(*)$ & Macrocheles pa lo skii $(* * *)$ \\
Dendrolaelaps strenzkei $(* *)$ & Macrocheles ernalis $(* * *)$ \\
Parasitus consanguineus $(*)$ & Haemogamarus pontiger $(* *)$ \\
Parasitus hyalinus $(*)$ & Parasitus talparum $(*)$ \\
& Uroobo ella crenelata $(* * *)$ \\
CERDO & GALLINA \\
Macrocheles merdarius $(* * *)$ & Holotaspis heterosetosa $(* * *)$ \\
Ameroseius plumosus $(* *)$ & Amblysenius obtusus $(* *)$ \\
Ameroseius delicatus $(* * *)$ & Rhodacarus coronatus $(* *)$ \\
Gamasodes bispinosus $(* * *)$ & Uroseius degeneratus $(* *)$ \\
CABALLO & Trichouropoda posnaniensis $(* *)$ \\
Macrocheles insignitus $(* * *)$ & Trichouropoda o alis $(* *)$ \\
Dendrolaelaps stammeri $(*)$ & Trichouropoda longio alis $(* * *)$ \\
Pergamasus agabundus $(* * *)$ & Nenteria bre iunguiculata $(* *)$ \\
Parasitus eta $(*)$ & Discourella cordieri $(* * *)$ \\
Nenteria floralis $(* * *)$ & \\
Nenteria stammeri $(* * *)$ & \\
Uroobo ella difo eolata $(* * *)$ & \\
Uroobo ella arians $(* * *)$ & \\
\hline
\end{tabular}

Tabla 6. Acaros gamásidos, bioindicadores de diferentes tipos de excrementos. Los asteriscos indican la calidad bioindicadora (* buena; ** muy buena; *** excelente) de los distintos taxones (tomado de Schelvis, 1992).

llega hasta el punto de poder identificar suelos quemados incluso tras noventa años de haberse producido la agresión (Athias-Binche, 1987).

\section{A MODO DE CONCLUSIÓN}

Evidentemente, los breves comentarios realizados en este trabajo no pueden proporcionarnos un cuadro someramente representativo de los grupos de fauna bioindicadora de espacios agrarios (por ejemplo, micromamíferos, insectos, moluscos terrestres, isópodos, etc.) aunque, al menos, confiamos en que estas pinceladas sirvan para proporcionar un marco de referencia de cara a emprender actuaciones operativas en el futuro.

Durante quizás demasiado tiempo la arqueozoología ha sido la "ciencia de los huesos" o, secundariamente, de las conchas. Pero ni unos ni otras agotan el amplio repertorio del mundo animal de nuestros antepasados, ni siquiera en relación con el proceso de la domesticación. Por esta razón, convendría empezar a llevar a cabo un repaso sistemático de la fauna y percatarse de que muchos otros animales han podido ser recursos fundamentales 
en determinados tipos de economías (por ejemplo, crustáceos) y que son, sobre todo, los taxones desconocidos, los insignificantes, los microscópicos, aquellos que nos permitirán alcanzar eventualmente la fiabilidad en las reconstrucciones paleoambientales que la fauna "clásica" nos niega sistemáticamente. Sólo ante la admisión de esta circunstancia y de nuestro profundo desconocimiento de sus posibilidades podremos ser capaces de concebir una arqueozoología más integradora.

\section{AGRADECIMIENTOS}

El texto corresponde a la comunicación "La fauna como bioindicadora de espacios agrarios en la Prehistoria" con la que participé en las Jornadas sobre Arqueología de los Espacios Agrarios (Salobreña, Granada, octubre de 1993), organizadas por la Universidad de Granada y el Excmo. Ayuntamiento de Salobreña.

Este trabajo ha sido realizado con una subvención de la DGICYT (PB 94-0186).

\section{BIBLIOGRAFÍA}

Anderson, E. (1956): "Man as a Maker of new Plants and new Plant Communities". In W.L. Thomas (ed.): "Man's role in changing the face of the Earth". Chicago University Press. Chicago: 763-777.

Arillo, A.; Gil-Martín, J. y Subías, L.S. (1992): “Acaros oribátidos subfósiles de Galicia". Boletín Sociedad Portuguesa de Entomología, Suplemento 4.

Athias-Binche, F. (1987): "Modalités de la cicatrisation des ecosystèmes méditerranéens aprés incendie: cas de certain arthropodes du sol. 3. Les Acariens Uropodides". Vie et Milieu, 37(1): 39-52.

BAIRD, R.F. (1989): "Fossil bird assemblages from australian caves: precise indicators of late Quaternary Environments?". Paleogeography, Paleoclimatology, Paleoecology, 69: 241-244

BERNIS, F. (1988): El medio urbano como receptor de fauna. En F. Bernis (ed.): Aves de los medios urbano y agrícola en las Mesetas Españolas. Publicaciones Sociedad Española de Ornitología (Monografías 2). Madrid: 7-26.

BoEv, Z. (1993): "Archaeo-ornithology the synanthropisation of birds: a case study for Bulgaria". Archaeofauna, 2: $145-153$

Gil-Martín, J. y Subías, J.L. (1995): "El estudio de los ácaros Oribátidos en España: estado actual del conocimiento y su utilización como bioindicadores edáficos".
En Historia Natural'93. Instituto de Estudios Alto Aragoneses. Jaca: 383-392.

Hernández, A. y Alegre, J. (1991): "Las aves de los setos en la provincia de León. Quercus, 64: 22-24.

IANKOV, P. (1983): "On the stages and criteria of synanthropisation in birds". Biologicheskie osnovy osvoenija, reconstruktsii $i$ ochrany Zhivotnogo mira Belorusii: 133-134.

IVERSEN, J. (1956): "Forest clearance in the Stone Age. Scientific American, 194(3): 320-326.

JuANA, E. de (1989): Las aves esteparias en España. Seminario sobre zonas áridas en España. Publicaciones Real Academia de Ciencias Exactas, Físicas y Naturales. Madrid: 199-221.

Krivolutsky, D.A. \& DRUK, A.Y. (1986): "Fossil oribatid mites". Annual Review of Entomology, 31: 533- 545.

Mahmud, M.A.; Mínguez, M.E. y SubíAs, J.L. (1984): "Estudio taxocenótico de los oribátidos (Acarida) de una zona agrícola de Toledo". Actas I Congreso Ibérico de Entomología (León, 20-junio-1983). Publicaciones Universidad de León. León: 459-470.

MAISELS, C.H. (1990): "The Emergence of Civilization". Routledge. London.

MÉNA, J.; LumbrerAS, C.J. y Rojo, S. (1990): "La boñiga: un ecosistema en miniatura". Quercus, 51: 39-43.

Morales, A. (1989): “Alteraciones antrópicas de las poblaciones animales naturales". $1 .{ }^{\text {as }}$ Jornadas Hombre y Medio Ambiente (Alcobendas, 11-12 octubre 1988). Ayuntamiento de Alcobendas, Madrid: 125-137.

- (1993): "Ornithoarchaeology: the various aspects of the classification of bird remains from archaeological sites". Archaeofauna, 2: 1-13.

Morales, A. y SANZ, J.L. (1994): “Arqueoacarología: Potencialidades y limitaciones de una prácticamente inédita subdisciplina arqueozoológica”. Pyrenae, 25: 1729.

— (e.p.): "La fauna del poblado de Peñalosa (prov. Jaén). Síntesis arqueozoológica". Noticiario Arqueológico de Andalucía.

O'Connor, R.J. \& SchrubB, M. (1986): “Farming and Birds". Cambridge University Press. Cambridge.

PARra, F. (1982)a: "La dehesa/1. El equilibrio del hombre con la naturaleza". Quercus, 2: 15-18.

- (1982)b: "La dehesa/2. La transformación del bosque mediterráneo". Quercus, 3: 45-47.

- (1982)c: "Los setos: naturaleza en un mínimo espacio". Quercus, 6: 61-63.

Ruiz, E.; Mínguez, M.E. y Subías, L.S. (1986): "Los oribátidos (Acari, Oribatida) de los eriales de cultivo de una zona agrícola del sur de Madrid y el efecto borde". Actas VIII Jornadas Asociación Española de Entomología (Sevilla, 29 septiembre-3 octubre 1986). Publicaciones Universidad de Sevilla, Sevilla: 98-110.

Schelvis, J. (1990): "Mites (Acari) in Archaeology". Proceedings Experimental and Applied Entomology, 1: 90-95.

- (1992): "Mites and Archaeozoology". Tesis Doctoral. Universidad de Groningen. Groningen.

- (1993): Restos de ácaros como indicadores medioambientales en estudios de la Geología Cuaternaria y de la Paleozoología". Investigación, 30: 75-77. 
ShEAR, W.A.; Bonamo, P.M.; Grierson, J.D.; Rolfe, I.; SMITH, E.L. \& NORTON, R.A. (1984): "Early land animals in North America: evidence from Devonian age Arthropods from Gilboa, New York". Science, 224: 492-494.

Simmons, I.G. (1982): "Biogeografía Natural y Cultural". Omega. Barcelona.

SuÁreZ, F. y RAmíreZ, L. (1987): "Las comunidades de aves de las "estepas" ibéricas". Quercus, 26: 16-20.

Subías, L.S. y Mínguez, M.E. (1985): "Los Oppidos (Acari, Oribatida) de El Pardo (España Central). Serratopiia n. gen. y Oxyoppioides n. gen.”. Boletín Sociedad Portuguesa Entomología 1, Suplemento 1: 165-174.

SubíAs, L.S.; Ruiz, E. y Mínguez, M.E. (1985): “Aportación al conocimiento de las comunidades de oribátidos (Acari) del erial mediterráneo". Boletín Sociedad Portuguesa Entomología 1, Suplemento 1: 389-398.

- (1986): "Consideraciones generales sobre la sucesión y bioindicación en los oribátidos (Acari, Oribatidae) de un erial de cultivo mediterráneo. Actas VIII Jornadas Asociación Española Entomología (Sevilla, 29 septiembre-9 octubre 1986). Publicaciones Universidad de Sevilla. Sevilla: 121-131.
Subías, L.S.; Arillo, A. y Gil-Martín, J. (1992): "Consideraciones biogeográficas sobre los oribátidos (Acari, Oribatida) de Marruecos y SÁhara Occidental”. En A. Alemany (ed.): Historia Natural 91. Ed. Bilbilis. Palma de Mallorca: 189-202.

TCHERnOv, E. (1992): "Evolution of complexities. Exploitation of the biosphere and zooarchaeology". Archaeozoologia, $\mathrm{V}(1)$ : 9-42.

Tellería, J.L.; SAntos, T.; Álvarez, G. y Sáez-RoyueLA, C. (1988): "Avifauna de los campos de cereales del interior de España”. En F. Bernis (ed.): Aves de los medios urbano y agrícola en las Mesetas Españolas. Publicaciones de la Sociedad Española de Ornitología (Monografías 2), Madrid: 173-317.

VAlVERdE, J.A. (1958): "Las aves esteparias de la Península Ibérica”. Publicaciones Instituto de Biología Aplicada 27: 41-48.

WhitTAKer, R.H. \& LiKENs, G.E. (1975): "The Biosphere and man. In H. Lieth \& R.H. Whittaker (eds.): "Primary productivity of the biosphere". Springer Verlag. Berlín: 305-318. 